\title{
Intranasal Poly-IC treatment exacerbates tuberculosis in mice through the pulmonary recruitment of a pathogen-permissive monocyte/macrophage population
}

\author{
Lis R.V. Antonelli, ${ }^{1}$ Antonio Gigliotti Rothfuchs, ${ }^{1}$ Ricardo Gonçalves, ${ }^{2}$ Ester Roffê, ${ }^{3}$ \\ Allen W. Cheever, ${ }^{1}$ Andre Bafica, ${ }^{4}$ Andres M. Salazar, ${ }^{5}$ Carl G. Feng, ${ }^{1}$ and Alan Sher ${ }^{1}$ \\ ${ }^{1}$ Laboratory of Parasitic Diseases, National Institute of Allergy and Infectious Diseases (NIAID), NIH, Bethesda, Maryland, USA. \\ 2Department of Cell Biology and Molecular Genetics, University of Maryland, College Park, Maryland, USA. ${ }^{3}$ Laboratory of Molecular Immunology, \\ NIAID, NIH, Bethesda, Maryland, USA. 4Department of Microbiology, Immunology and Parasitology, \\ Federal University of Santa Catarina, Florianopolis, Brazil. ${ }^{5}$ Oncovir Inc., Washington, DC, USA.
}

\begin{abstract}
Type I IFN has been demonstrated to have major regulatory effects on the outcome of bacterial infections. To assess the effects of exogenously induced type I IFN on the outcome of Mycobacterium tuberculosis infection, we treated pathogen-exposed mice intranasally with polyinosinic-polycytidylic acid condensed with poly-L-lysine and carboxymethylcellulose (Poly-ICLC), an agent designed to stimulate prolonged, high-level production of type I IFN. Drug-treated, M. tuberculosis-infected WT mice, but not mice lacking IFN- $\alpha \beta$ receptor 1 (IFN $\alpha \beta R$; also known as IFNAR1), displayed marked elevations in lung bacillary loads, accompanied by widespread pulmonary necrosis without detectable impairment of Th1 effector function. Importantly, lungs from Poly-ICLC-treated M. tuberculosis-infected mice exhibited a striking increase in $\mathrm{CD}_{11 \mathrm{~b}} \mathrm{~F}^{+} / \mathbf{8 0}^{+} \mathrm{Gr} 1^{\text {int }}$ cells that displayed decreased MHC II expression and enhanced bacterial levels relative to the same subset of cells purified from infected, untreated controls. Moreover, both the Poly-ICLC-triggered pulmonary recruitment of the $\mathrm{CD} 11 \mathrm{~b}^{+} \mathrm{F} 4 / \mathrm{80}^{+} \mathrm{Gr} 1^{\text {int }}$ population and the accompanying exacerbation of infection correlated with type I IFN-induced upregulation of the chemokine-encoding gene $\mathrm{Ccl} 2$ and were dependent on host expression of the chemokine receptor CCR2. The above findings suggest that Poly-ICLC treatment can detrimentally affect the outcome of $M$. tuberculosis infection, by promoting the accumulation of a permissive myeloid population in the lung. In addition, these data suggest that agents that stimulate type I IFN should be used with caution in patients exposed to this pathogen.
\end{abstract}

\section{Introduction}

Type 1 IFNs were described more than fifty years ago and were named based on their ability to interfere with virus infection (1). All type I IFNs are recognized by a single type I IFN receptor, IFN $\alpha \beta$ r. IFN- $\alpha$ and IFN- $\beta$ are the 2 main subtypes of type I IFN of immunological interest and both are rapidly induced after exposure to many viruses as well as other infectious agents (2-4). In addition to their role in control of viral replication, type I IFNs are known to perform a number of important regulatory functions, affecting both the innate and adaptive immune response. These include the activation of APCs and NK cells as well as the enhancement of $\mathrm{CD}^{+} \mathrm{T}$ lymphocyte proliferation and differentiation of Th1 cells through their agonist effects on IL-12 production (5). In different settings, type I IFN can also exert downregulatory effects on important immune functions, such as APC activation, and can suppress $\mathrm{T}$ cell proliferation and hematopoiesis $(6,7)$.

In addition to their well-documented roles in the host response to viral pathogens, type I IFNs have more recently been demonstrated to have major regulatory effects on the outcome of bacterial infection. Thus, mice genetically impaired in type I IFN signaling display enhanced resistance to Listeria monocytogenes, an effect attributed to

Conflict of interest: Andres M. Salazar is Chief Executive Officer and Scientific Director of Oncovir Inc., which provides Poly-ICLC.

Citation for this article: J Clin Invest. 2010;120(5):1674-1682. doi:10.1172/JCI40817. the loss in immune cell death normally mediated by type I IFN in WT animals $(8,9)$. In mycobacterial infection, both protective and deleterious effects of type I IFN have been described. In Mycobacterium avium infection, the continuous infusion of IFN- $\beta$ led to increased resistance as evidenced by a $1-\log$ reduction in hepatic and splenic bacterial loads (10). However, in other studies involving murine M. tuberculosis infection, endogenous type I IFN appeared to promote rather than limit bacterial growth (11-13). Thus, Manca and collaborators correlated the hypervirulence of a $M$. tuberculosis strain with enhanced type I IFN synthesis, which was associated with impaired Th1 immune responses (11). Later, it was shown that type I IFN receptor-deficient mice, chronically infected with a variety of different $M$. tuberculosis strains, displayed significantly reduced bacterial loads when compared with similarly infected WT animals (13).

Type I IFN has been demonstrated to have therapeutic efficacy in a number of viral infections and malignancies $(14,15)$. Given the above findings documenting both positive and negative effects of type I IFN on host resistance to mycobacteria, it was of interest to determine whether exogenous induction of these cytokines during M. tuberculosis infection would be host beneficial or detrimental. To address this question, we studied the effects on murine tuberculosis of polyinosinic-polycytidylic acid condensed with poly-L-lysine and carboxymethylcellulose (Poly-ICLC), a stabilized version of Poly-IC currently used in clinical trials because of its long half-life and potent induction of persistent type I IFN responses $(16,17)$. 
A

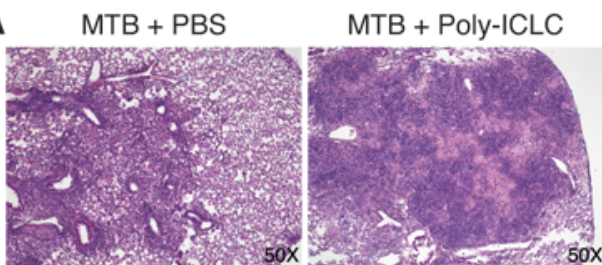

C

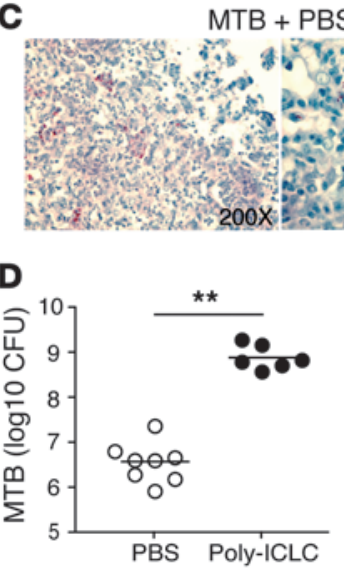

E

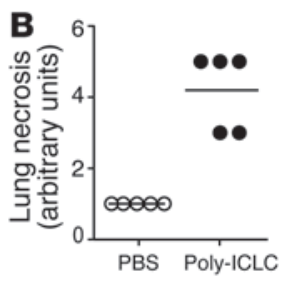

MTB + Poly-ICLC
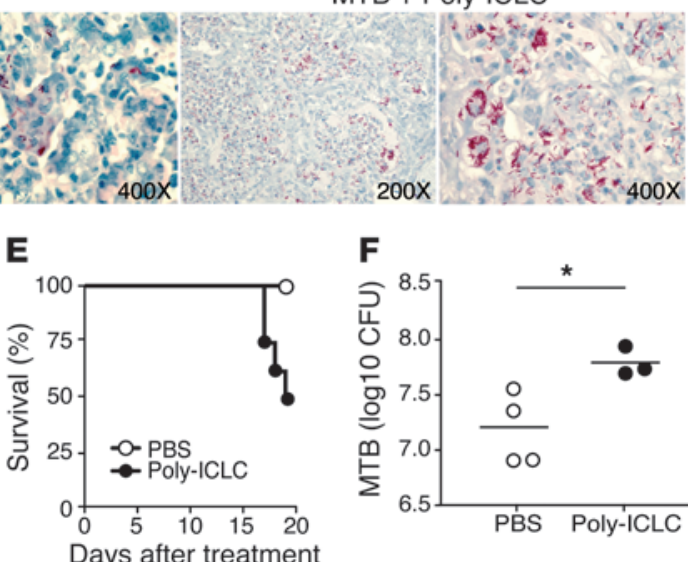

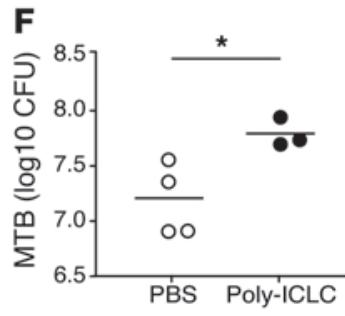

Figure 1

Poly-ICLC treatment increases host susceptibility to acute and chronic $M$. tuberculosis infection in mice. WT mice were infected with $M$. tuberculosis (MTB) by aerosol and treated with Poly-ICLC, starting either 1 day $(\mathbf{A}-\mathbf{D})$ or 4 months ( $\mathbf{E}$ and $\mathbf{F})$ after exposure. (A) Representative images of H\&E-stained lung sections and (B) histopathological evaluation of pulmonary necrosis in Poly-ICLC- or PBS-treated mice, 4 weeks after initiation of treatment of acutely infected mice. (C) Acid-fast staining and (D) bacterial load in the lungs of Poly-ICLC- or PBS-treated animals. (E) Survival and (F) pulmonary mycobacterial loads in Poly-ICLC- or PBS-treated mice $(n=5)$ exposed to $M$. tuberculosis 4 months earlier. The analysis was performed 3 weeks after treatment, due to increased mortality in drug-treated animals. Filled and open circles represent Poly-ICLC- or PBStreated animals, respectively. In $\mathbf{B}, \mathbf{D}$, and $\mathbf{F}$, circles indicate individual mice. Horizontal lines represent comparisons between Poly-ICLC- and PBS-treated, $M$. tuberculosis-infected mice. The data shown are representative of $5(\mathbf{A}-\mathbf{D})$ and $2(\mathbf{E}$ and $\mathbf{F})$ independent experiments. Original magnification, $\times 50(\mathbf{A})$; $\times 200$ (C, first and third panels); $\times 400$ (C, second and fourth panels). ${ }^{*} 0.01<P$ value $<0.05,{ }^{* *} P<0.01$.
As described in this study, we observed a major exacerbating effect of Poly-ICLC on both lung pathology and pulmonary bacterial load in M. tuberculosis-infected mice. Interestingly, this disease outcome was closely associated with a striking alteration in the pulmonary myeloid compartment and in particular with the CCR2-dependent infiltration of a monocyte/macrophage subpopulation highly permissive for bacterial growth.

\section{Results}

Intranasal Poly-ICLC treatment exacerbates pulmonary pathology and enhances bacterial growth in murine M. tuberculosis infection. To evaluate the effect of Poly-ICLC on M. tuberculosis infection, mice were exposed to a low dose of $\mathrm{H} 37 \mathrm{Rv}$ strain bacteria via aerosol and treated intranasally twice weekly with the drug, beginning on the day after infection. When examined on the fourth week, the infected mice treated with Poly-ICLC showed marked alterations in pulmonary pathology compared with infected, PBS-treated animals. While lung sections from both groups displayed mononuclear cell infiltration and numerous granulomas, the drug-treated mice exhibited extensive areas of necrosis (Figure 1, A and B). In addition, lungs from the PolyICLC-treated mice showed an approximately 2-log increase in bacterial load relative to PBS-treated animals (Figure 1, $\mathrm{C}$ and D). Drug-treated, infected mice also displayed significant weight loss (data not shown). In contrast, no pathological changes were detected in noninfected mice treated with PolyICLC under the same regimen.

In a separate series of experiments, drug treatment was initiated during chronic infection (4 months after aerosol exposure). In this situation, mortality of the infected mice was observed as early as 17 days after Poly-ICLC treatment (Figure 1E), and the remaining euthanized mice again showed a similar, although not as marked, increase in pulmonary bacillary burden (Figure 1F), possibly due to the shorter duration of drug administration.
Poly-ICLC-induced exacerbation of murine tuberculosis is dependent on type I IFN. As expected, Poly-ICLC administration resulted in the induction of high levels of Ifnb and Ifna mRNA as well as IFN- $\beta$ protein in the lungs of both uninfected and M. tuberculosis-exposed animals (Figure 2, A-C). To assess whether type I IFN stimulation is responsible for the changes in pulmonary pathology and bacterial load seen in Poly-ICLC-treated animals, Ifnabr-deficient mice were infected and treated using the same regimen. While PBS-treated, Ifnabr-deficient mice showed higher bacterial burdens than WT animals, no further increase in pulmonary bacillary load or pathology was seen after Poly-ICLC administration (Figure 2, D and E). These results confirmed that exacerbation of tuberculosis by PolyICLC is dependent on type I IFN signaling.

The effector $T$ cell compartment in M. tuberculosis-infected mice is not altered as a consequence of type I IFN induction. We next examined whether Poly-ICLC treatment exacerbates murine tuberculosis by directly affecting $\mathrm{CD}^{+} \mathrm{T}$ cell activation and differentiation, the mechanism suggested for the effects of endogenous type I IFN in promoting the growth of hypervirulent $M$. tuberculosis clinical isolates (12). Interestingly, Poly-ICLC administration did not affect the accumulation of $\mathrm{CD}_{4} 4^{+}$(Figure $3 \mathrm{~A}$ ) or $\mathrm{T}$ box expressed in $\mathrm{T}$ cells (T-bet) $\mathrm{CD} 4^{+}$ $T$ cells (Figure 3B) in the lungs of $M$. tuberculosis-infected mice. Similarly, both Poly-ICLC- and PBS-treated animals displayed indistinguishable frequencies of IFN- $\gamma$ and TNF- $\alpha$ double-producing CD4 ${ }^{+}$ T cells following ex vivo restimulation with anti-CD3, purified protein derivative (PPD) (Figure 3C), or peptide epitopes from $M$. tuberculosis antigens ESAT- 6 or Ag85B (data not shown). Furthermore, no significant differences between Poly-ICLC- and PBS-treated groups were evident when IFN- $\gamma$ levels were measured by ELISA in supernatants from the same pulmonary leukocyte cultures (Supplemental Methods and Supplemental Figure 2; supplemental material available online with this article; doi:10.1172/JCI40817DS1). Together, these observations suggest that the exacerbation of tuberculosis by Poly-ICLC is not the result of altered Th1 effector function. 

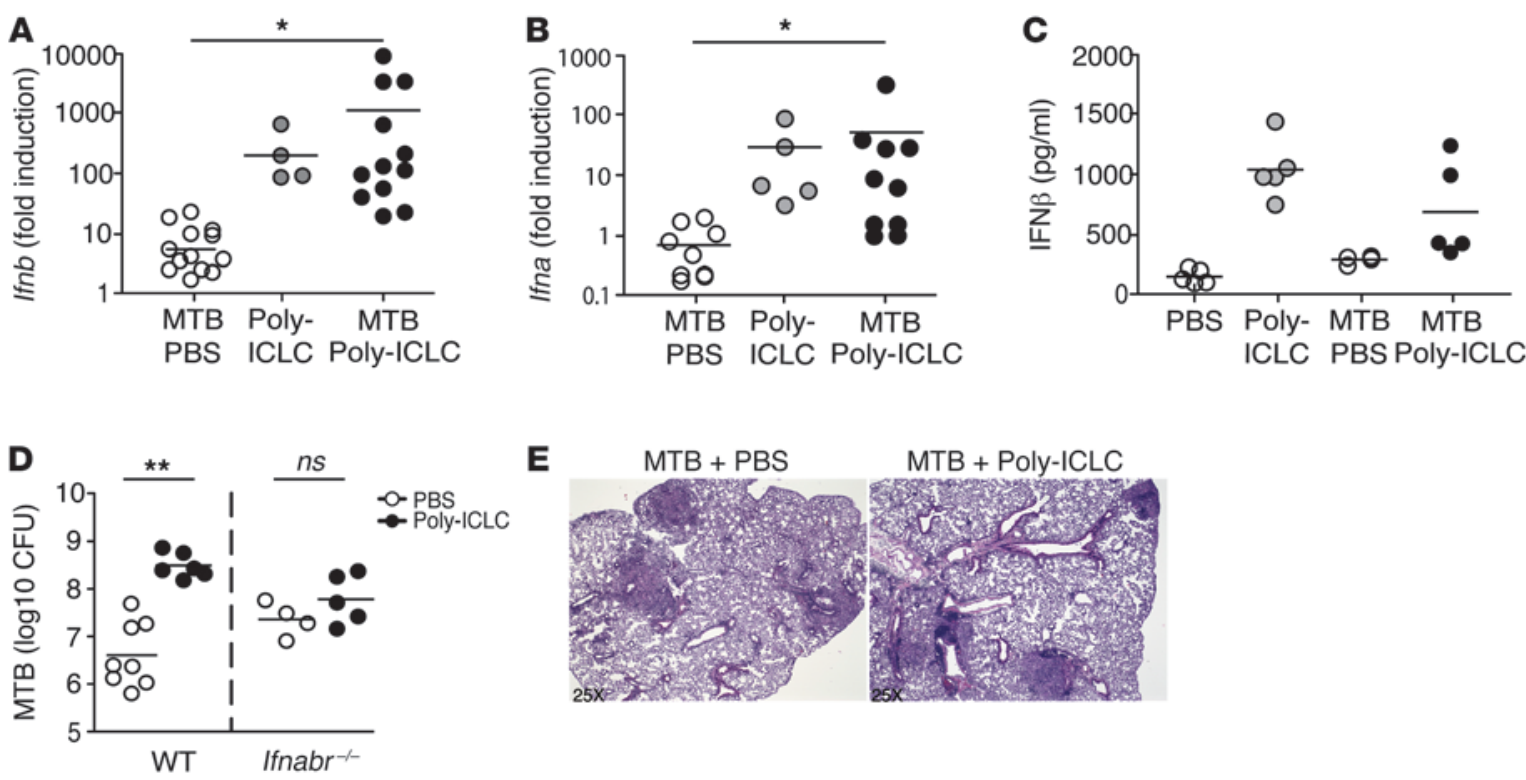

E

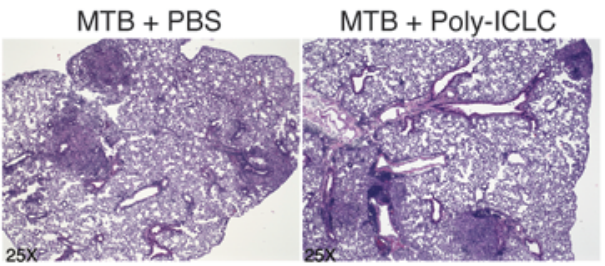

Figure 2

Poly-ICLC-triggered exacerbation of murine tuberculosis is dependent on type I IFN signaling. (A) Ifnb and (B) Ifna (all genes) mRNA expression levels, determined by real-time PCR in the lungs of $M$. tuberculosis-infected animals (open circles), naive mice treated with Poly-ICLC (gray circles), and M. tuberculosis-infected animals treated with Poly-ICLC (filled circles). Results are presented as the fold increase relative to transcript levels in PBS-treated, naive mice. Data are pooled from 3 independent experiments with similar results. (C) IFN- $\beta$ protein levels determined by ELISA in lung homogenates of naive and M. tuberculosis-infected animals, PBS (open circles) or Poly-ICLC-treated (filled circles). (D) Mycobacterial loads in PBS-treated (open circles) or Poly-ICLC-treated (filled circles) WT and Ifnabr-1- mice. (E) H\&E-stained lung tissue sections demonstrating indistinguishable pulmonary pathology between Poly-ICLC- and PBS-treated, M. tuberculosis-infected Ifnabr ${ }^{-1-}$ mice $^{-}$Circles indicate individual mice throughout. Results are representative of 3 independent experiments. Original magnification, $\times 25$ (E). (A-D) Horizontal lines represent comparisons between Poly-ICLC- and PBS-treated, M. tuberculosis-infected mice. ${ }^{\star} 0.01<P$ value $<0.05$, ${ }^{\star \star} P<0.01$.

Poly-ICLC triggers major changes in the composition of the myeloid compartment in lungs of M. tuberculosis-infected mice. Since no impairment in Th1 immune response was observed in Poly-ICLC-treated, infected mice, we next asked whether the exacerbation of disease is associated with changes in myeloid effector cells. To investigate this, the CD3- lung population was analyzed for the expression of CD11b, Gr1, F4/80, CD115, CD68, and MAC-3 as well as other markers (Figure 4). A major increase in both the frequencies (mean \pm SEM, $37.17 \% \pm 3.99 \%$ versus $3.81 \% \pm 1.98 \%$; Figure $4 \mathrm{~A}$ ) and the absolute numbers (Figure $4 \mathrm{~B}$ ) of pulmonary $\mathrm{CD} 11 \mathrm{~b}^{+} \mathrm{Gr} 1^{\text {int }}$ cells was observed in the M. tuberculosis-infected and drug-treated animals relative to the PBStreated, infected group. Annexin staining experiments suggested that the altered distribution of $C D 11 b^{+} G r 1^{\text {int }}$ versus $C D 11 b^{+} G r 1^{-}$cells in the lungs of mice receiving Poly-ICLC can be accounted for in part by

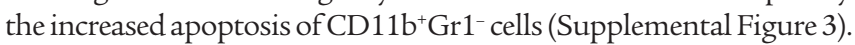
In addition, the Poly-ICLC-treated, infected mice showed higher frequencies of cells expressing the monocyte/macrophage markers F4/80, CD68, MAC-3, and CD115 (Figure 4C).

To confirm that the observed changes in the pulmonary myeloid compartment are due to induction of type I IFN by PolyICLC, the above experiments were performed in Ifnabr-deficient mice. In direct contrast to results seen in WT mice, no increases in the frequencies and absolute numbers of CD $11 \mathrm{~b}^{+} \mathrm{Gr} 1^{\text {int }}$ cells were observed when these animals, incapable of type I IFN signaling, were infected and drug treated (Figure 5, A and B). In the same experiments, we observed that Poly-ICLC treatment induced a substantial decrease in the frequency of MHC class II-expressing monocyte/macrophages (F4/80+ cells), which was not seen in infected, drug-treated Ifnabr-deficient mice (Figure 5, $A$ and $B)$. Consistent with this finding, IFN- $\alpha 4$ and IFN- $\beta$ inhibited the upregulation of MHC class II, mediated by IFN- $\gamma$ in M. tuberculosis-infected bone marrow-derived macrophages (Supplemental Methods and Supplemental Figure 4). Together, the above observations indicated that Poly-ICLC treatment results in a profound type I IFN-dependent alteration in the composition of the pulmonary myeloid compartment in M. tuberculosisinfected mice that manifests as a major increase in $\mathrm{CD} 11 \mathrm{~b}^{+} \mathrm{Gr} 1^{\text {int }}$ $\mathrm{F} 4 / 80^{+} \mathrm{MHCII}{ }^{\text {low }}$ cells.

The pulmonary $C D 11 b^{+} G r 1^{\text {int }}$ myeloid population induced by PolyICLC treatment preferentially supports growth of $M$. tuberculosis in vivo. In addition to their increased frequency, it was possible that the $\mathrm{CD} 11 \mathrm{~b}^{+} \mathrm{Gr} 1^{\text {int }}$ myeloid cells appearing in the lungs of infected Poly-ICLC-treated mice were more permissive to bacterial growth. To test this hypothesis, we FACS-sorted the $\mathrm{CD} 11 \mathrm{~b}^{+} \mathrm{Gr} 1^{-}$and $\mathrm{CD} 11 \mathrm{~b}^{+} \mathrm{Gr} 1^{\text {int }}$ subpopulations from infected, PBS- and drug-treated animals, 4 weeks after infection, and plated equal numbers of each on 7H11 agar to measure their pathogen content (Figure 6). Although bacterial growth was seen in the $\mathrm{CD} 11 \mathrm{~b}^{+} \mathrm{Gr} 1^{-}$cell subpopulation, the number of colonies per host cell did not differ between PBS- and drug-treated animals (Figure 6). In direct contrast, PolyICLC treatment resulted in a 10 -fold increase in bacterial growth in $\mathrm{CD} 11 \mathrm{~b}^{+} \mathrm{Gr} 1^{\text {int }}$ cells purified from drug-treated animals compared with the same population recovered from control mice (Figure 6). These observations indicate that the pulmonary $\mathrm{CD} 11 \mathrm{~b}^{+} \mathrm{Gr} 1^{\text {int }}$ cell subpopulation, elevated as a result of type I IFN induction, preferentially supports mycobacterial expansion/survival. 

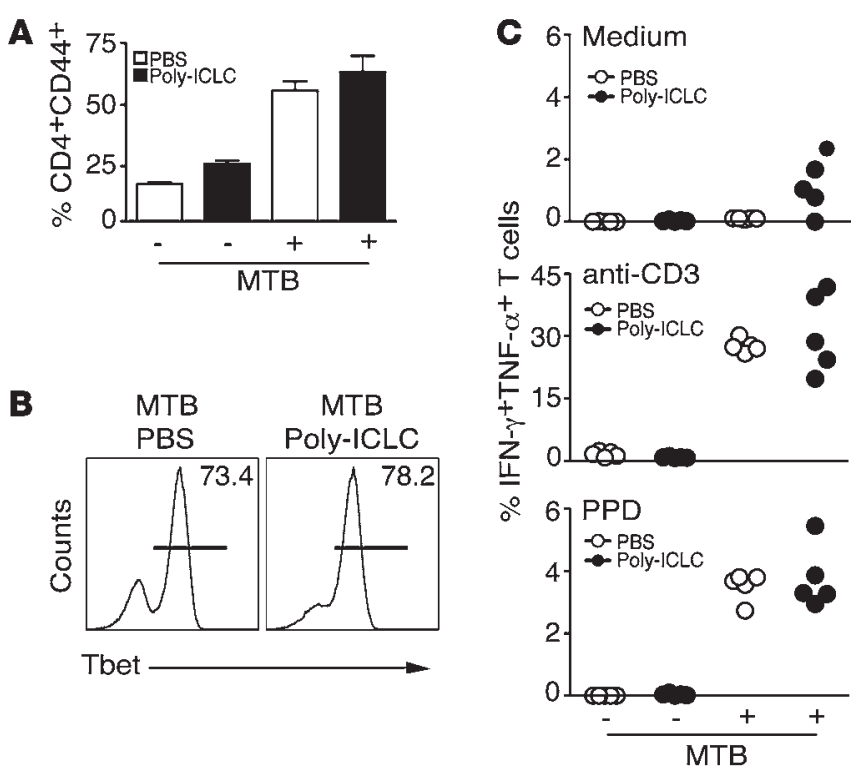

Impaired intracellular control of $M$. tuberculosis has been associated with the Th2 cytokine-dependent generation of alternatively activated macrophages and inhibition of autophagic microbicidal activity $(18,19)$ and thus could represent a possible explanation for the effects of Poly-ICLC on macrophage function described here. Although no increase in Il4 mRNA expression was evident in the lungs of drug-treated, infected mice (data not shown), a significant elevation in Il13 transcripts was observed (Supplemental Figure 5A). Nevertheless, Poly-ICLC treatment of Il13ra1-deficient mice led to increases in pulmonary bacterial load and $\mathrm{CD} 11 \mathrm{~b}^{+} \mathrm{Gr} 1^{\text {int }}$ cell infiltration that were indistinguishable from those observed in WT animals (Supplemental Figure 5, B and C).

An additional hypothesis tested was that Poly-ICLC treatment results in decreased macrophage IFN- $\gamma$ receptor expression, leading to defective induction of microbicidal activity mediated by that cytokine. Indeed, the $\mathrm{CD} 11 \mathrm{~b}^{+} \mathrm{Gr} 1^{\text {int }}$ cell population from the lungs of infected, drug-treated mice displayed significantly decreased IFN- $\gamma$ receptor expression compared with untreated, infected animals. Nevertheless reductions in IFN- $\gamma$ receptor expression were also observed in the $\mathrm{CD}_{11} \mathrm{~b}^{+} \mathrm{Gr} 1^{-}$population, which failed to show increased bacterial loads following Poly-ICLC treatment (Supplemental Figure 6). Moreover, no difference in Nos2 message and NOS2 protein expression were detected in the CD $11 \mathrm{~b}^{+} \mathrm{Gr} 1^{\text {int }}$ cell population recovered from control versus drug-treated infected mice (Supplemental Figure 7). Together, the above findings suggest that decreased monocyte/macrophage IFN- $\gamma$ receptor expression, while possibly contributing to the enhanced bacterial growth seen in the lungs of Poly-ICLC-treated mice, cannot be in itself the major mechanism underlying this outcome.

The impaired control of M. tuberculosis infection triggered by Poly-ICLC treatment is CCR2 dependent. We next addressed the mechanism responsible for accumulation of the $\mathrm{CD} 11 \mathrm{~b}^{+} \mathrm{Gr} 1^{\text {int }}$ cell subpopulation in the lungs of infected mice, treated with Poly-ICLC. To do so, we examined the expression, using real-time PCR in wholelung tissue, of a set of chemokine and chemokine receptor genes known to be involved in myeloid cell recruitment (Figure 7A). No significant differences were observed in the expression of Cxcl1 and its receptor $C x c r 2$ in the same 2 groups of animals. In contrast,

\section{Figure 3}

Poly-ICLC treatment does not result in suppressed Th1 effector function in infected animals. Naive or M. tuberculosis-infected WT mice were treated with PBS (open symbols) or Poly-ICLC (filled symbols) for 4 weeks, starting 1 day after pathogen exposure. (A) Frequency of CD4 ${ }^{+}$CD $44^{+} \mathrm{T}$ cells in pulmonary leukocyte populations isolated from naive or M. tuberculosis-infected animals treated with PBS or PolyICLC. The data shown are the mean $\pm \operatorname{SEM}(n=5)$. (B) Frequency of

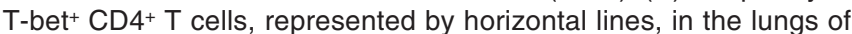
PBS- or drug-treated, infected animals. (C) Frequency of $\mathrm{CD} 4{ }^{+} \mathrm{CD} 44^{+}$ $\mathrm{T}$ cells producing IFN- $\gamma$ and TNF- $\alpha$ in response to in vitro stimulation with medium, anti-CD3, or PPD, determined by flow cytometry. Circles indicate individual animals. The data shown are representative of 3 independent experiments.

we observed an approximately 10 -fold enhancement in transcripts encoding Ccl2 in lungs of Poly-ICLC- versus PBS-treated, infected animals. This induction was dependent on type I IFN signaling, since no changes were observed when infected Ifnabr-deficient mice were treated with Poly-ICLC.

CCL2 expression has previously been associated with monocytic infiltration in several inflammatory diseases, and studies in Ccr2-deficient mice have demonstrated a major role for this chemokine receptor in the emigration of monocytes and macrophages from bone marrow and their recruitment to tissue sites at which CCL2 is expressed $(20,21)$. To determine whether the increased expression of $\mathrm{Ccl} 2$ in lungs of Poly-ICLC-treated, M. tuberculosis-infected mice reflects a function for this chemokine in recruitment of CD $11 \mathrm{~b}^{+} \mathrm{Gr} 1^{\text {int }}$ cells, we infected and drug-treated mice deficient in CCR2, the receptor for CCL2. The increased frequency of CD $11 b^{+} \mathrm{Gr} 1^{\text {int }}$ cells observed after Poly-ICLC treatment of infected WT mice was not seen in animals lacking CCR2 (Figure 7B). In agreement with previous studies (22), Ccr2-deficient mice displayed higher bacterial burdens than WT mice in the absence of drug treatment (Figure 7C). Importantly, however, drug treatment failed to induce an increase in bacterial load in the chemokine receptor knockout animals (Figure $7 \mathrm{C}$ ). Together these observations linked CCL2-CCR2-dependent recruitment of CD11b ${ }^{+} \mathrm{Gr} 1^{\text {int }}$ cells to the exacerbation of $M$. tuberculosis infection seen following Poly-ICLC administration.

\section{Discussion}

Tuberculosis threatens one-third of the world's population, and it is the leading cause of death by a single infectious agent. Cytokines such as IFN- $\gamma$ and TNF- $\alpha$ have been shown to play important functions in host resistance and/or immunopathology in $M$. tuberculosis infection and represent potential immunological intervention points in controlling this disease (23-25). Type I IFNs are a class of cytokines that play a major role in regulation of viral infection and have also been studied as possible mediators of immunity to other types of pathogens, including mycobacteria. Nevertheless, while positive effects of type I IFN in activating immune cells have been noted in several reports 


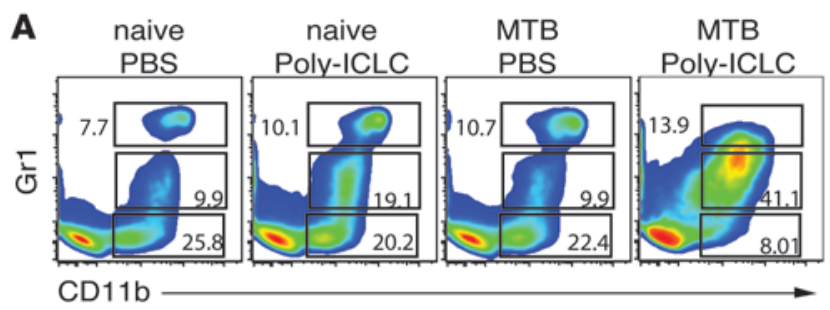

B
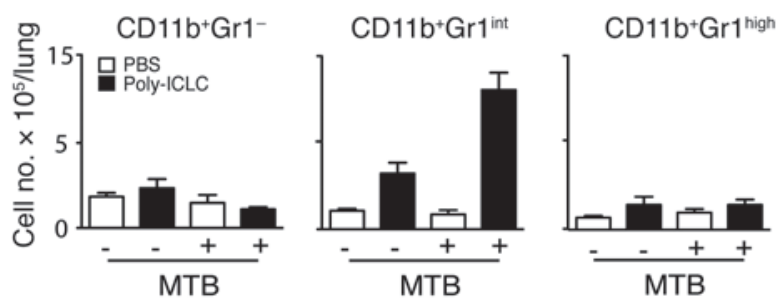

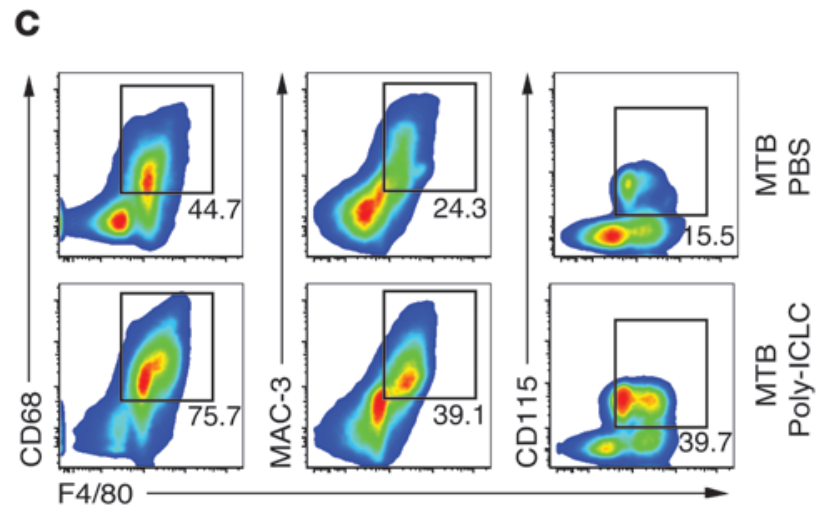

Figure 4

Poly-ICLC treatment triggers major alterations in pulmonary myeloid subsets in infected mice. (A) Representative flow cytometry dot plots showing the frequencies and (B) bar graphs indicating the mean total numbers ( \pm SEM) of CD11 b+Gr1-, CD11 b+Gr1 int, and CD11 b ${ }^{+}$rr $1^{\text {high }}$ pulmonary leukocytes isolated from naive or M. tuberculosis-infected animals treated with PBS (open bars) or Poly-ICLC (filled bars) $(n=5)$. The data shown are representative of 4 independent experiments. (C) Representative flow cytometry dot plots showing increased frequencies of F4/80-, CD115-, MAC-3-, and CD68-expressing cells in M. tuberculosis-infected mice after Poly-ICLC (bottom panels) or PBS administration (top panels). The data shown are representative of 3 independent experiments. (A and C) Numbers within or beside each box refer to the frequencies of cells within each gate.

examining M. tuberculosis infection in vitro, most previous studies have demonstrated deleterious influences of these cytokines on the outcome of infection with the pathogen in vivo (11-13). In the present report, we have addressed this issue by assessing the effects of a potent type I IFN inducer, Poly-ICLC, on murine M. tuberculosis. Our findings clearly establish that treatment with this drug dramatically exacerbates pulmonary immunopathology in aerosol M. tuberculosis infection, an outcome associated with type I IFN production and the CCR2-dependent recruitment of a monocyte/macrophage population, highly permissive for mycobacterial growth. The latter observation is consistent with recent findings, demonstrating a major role for macrophage dynamics in determining the fate of mycobacterial infection in vivo $(26,27)$.

As described here, intranasal administration of Poly-ICLC induced extensive pulmonary necrosis in mice infected with M. tuberculosis. Previous studies in the murine L. monocytogenes model have implicated a major role for both endogenous and exogenously induced type I IFN in triggering infection-induced immunopathology $(8,9)$. Thus, L. monocytogenes-infected WT mice but not Ifnabr-deficient mice display acute hepatic damage, and this response is greatly exacerbated when the WT mice are treated with Poly-IC. These pathological effects of type I IFN in vivo were consistent with earlier in vitro observations demonstrating that cytokine presensitized macrophages are more susceptible to L. monocytogenes-induced cell death (28).

In murine listeriosis, type I IFN induction is also associated with increased bacterial growth, which is likely to be a factor contributing to the observed pathology. Augmented pathogen expansion was also a major outcome in the experiments described here, in which a 2-log increase in pulmonary $M$. tuberculosis burden was seen in Poly-ICLC-treated, acutely infected mice. This finding is consistent with previous reports demonstrating a role for endogenous type I IFN in promoting $M$. tuberculosis infection (11-13). In the latter studies, a correlation between type I IFN-induced susceptibility

\section{Figure 5}

The alterations in pulmonary myeloid cell composition triggered by Poly-ICLC in infected mice are dependent on type I IFN signaling. Lung leukocytes were isolated from Poly-ICLC- and PBS-treated, M. tuberculosis-infected mice and analyzed by flow cytometry. (A) Representative dot plots and $(\mathbf{B})$ bar graphs showing mean frequencies ( \pm SEM) of CD11 b + Gr1 int (top panel) and $\mathrm{F} 4 / 80^{+} \mathrm{MHCll}^{+}$ (bottom panel) cells isolated from $M$. tuberculosis-infected WT mice (filled bars) or Ifnabr ${ }^{-1}$ animals (open bars), treated with PBS or Poly-ICLC $(n=4-5)$. The data shown are representative of 2 independent experiments Numbers beside each box refer to the frequencies of cells within each gate. ${ }^{*} 0.01<P$ value $<0.05$.
A
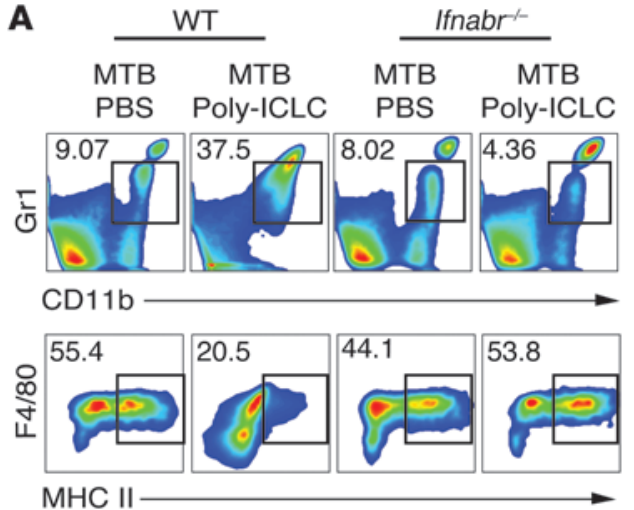

B

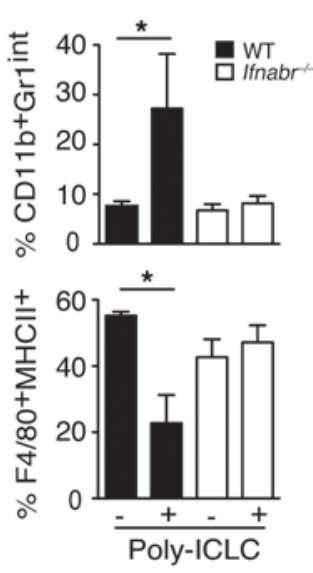



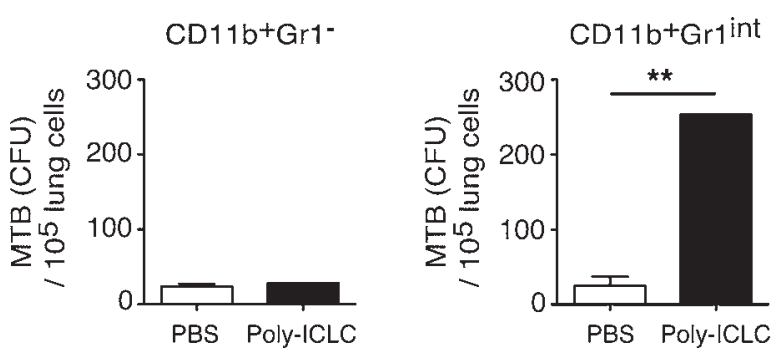

and decreased Th1 cytokine production was also documented and proposed as a possible explanation for the effects observed. In contrast, the exacerbation of H37Rv infection by Poly-ICLC treatment observed in our experiments was not associated with impaired Th1 response development, as assessed by the expression of T-bet and cytokine production by pulmonary $\mathrm{T}$ lymphocytes.

Subsequent experiments revealed that a major target of the effects of Poly-ICLC treatment on disease exacerbation was the myeloid cell compartment in the murine M. tuberculosis model. Thus, the lungs of drug-treated, infected mice exhibited a striking 4-fold increase in the frequency of $\mathrm{CD} 11 \mathrm{~b}^{+} \mathrm{Gr} 1^{\text {int }}$ cells, and the same was not observed when uninfected animals were treated with Poly-ICLC. This cell population was further characterized as belonging to the monocyte/macrophage lineage, based on the predominant expression of F4/80, MAC3, CD68, and CD115. Cells with a similar $\mathrm{CD} 115^{+} \mathrm{Gr} 1^{+}$phenotype have been described in a variety of inflammatory conditions and are usually characterized as inflammatory monocytes (29). Monocytes belonging to this subset undergo phenotypic and functional changes depending on their tissue environment, giving rise, for example, to TNF- $\alpha$ / iNOS-producing DCs in pathogen-infected mice (30) or to myeloid-derived suppressor cells in tumor-bearing hosts (31-33). In most infectious disease models, inflammatory monocytes have been associated with host defense rather than disease progression (29). For example, Gr1 $1^{+} \mathrm{F} 4 / 80^{+} \mathrm{CD} 11 \mathrm{~b}^{+} \mathrm{CD} 11 \mathrm{c}^{-}$inflammatory monocytes are recruited to the gut during peroral infection with Toxoplasma gondii and rescue the impaired resistance to the parasite seen in Ccr2-deficient mice (29).

Although infection with $M$. tuberculosis is known to dramatically alter monocyte differentiation in pulmonary tissue (34), the role of monocyte subsets in host resistance to the pathogen is poorly defined. As shown in the present study, the $\mathrm{CD} 11 \mathrm{~b}^{+} \mathrm{Gr} 1^{\text {int }}$ cell subset is not significantly expanded in the lungs of untreated, $M$. tuberculosis-infected mice. Nevertheless, these cells could contribute to bacterial control, as suggested by the impaired resistance to $M$. tuberculosis of Ccr2-deficient mice (22), which display defective migration of inflammatory monocytes from the bone marrow to the periphery (20).

While the above studies provide evidence for a protective role for inflammatory monocytes in M. tuberculosis infection, the data presented here suggest that, in the setting of upregulated type I IFN production, these cells can actually promote pathogen growth. Thus, the 4-fold expansion of $\mathrm{CD} 11 \mathrm{~b}^{+} \mathrm{Gr} 1^{\text {int }}$ cells seen in M. tuberculosis-infected mice treated with Poly-ICLC was accompanied by a major increase in bacterial burden, and these changes were not observed in drug-treated Ifnabr/- mice. Interestingly, in these experiments Ifnabr ${ }^{-/}$mice that were not treated with PolyICLC displayed significantly increase bacterial burdens, an effect not seen in previous studies on $M$. tuberculosis infection of these mice. Nevertheless, the increase observed was not accompanied by

\section{Figure 6}

The CD11b+Gr1 $1^{\text {int }}$ subpopulation in the lungs of Poly-ICLC-treated mice displays impaired control of $M$. tuberculosis. Pulmonary $\mathrm{CD} 11 \mathrm{~b}^{+} \mathrm{Gr} 1^{-}$and $\mathrm{CD} 11 \mathrm{~b}+\mathrm{Gr} 1^{\text {int }}$ cells were isolated by FACS from PolyICLC- and PBS-treated mice, 4 weeks after infection. The cells were then plated on agar, and bacterial CFUs were enumerated 3 weeks later. Bar graphs represent the mean \pm SEM of the bacterial counts per $10^{5}$ lung cells recovered from multiple mice $(n=4-6)$. The experiment shown is representative of 3 performed. ${ }^{* *} P<0.01$.

increased pulmonary pathology. Regardless of these findings with Ifnabr ${ }^{-1}$ mice, we cannot exclude the possibility that Poly-ICLC treatment has type I IFN-independent effects, as suggested in a previous study using synthetic dsRNA (35).

Importantly, cell-sorting experiments indicated that most of the increased bacterial burden triggered by Poly-ICLC can be accounted for in part by increased infection of the $\mathrm{CD} 11 \mathrm{~b}^{+} \mathrm{Gr} 1^{\text {int }}$ pulmonary monocyte/macrophage subpopulation. The latter finding suggested that Poly-ICLC treatment triggers expansion or recruitment to the lungs of an inflammatory monocyte population that is permissive to infection and fails to restrict bacterial growth. Interestingly, the $\mathrm{CD} 11 \mathrm{~b}^{+} \mathrm{Gr} 1^{\text {int }}$ cells accumulating in the lungs of Poly-ICLC-treated, infected mice showed decreased MHC class II expression relative to the same subset in infected, untreated controls. This observation is consistent with previous studies demonstrating that type I IFN antagonizes IFN- $\gamma$-induced MHC class II expression in macrophages in vitro (36), an outcome also seen here (Supplemental Figure 4). Poly-ICLC treatment also resulted in a decrease in IFN- $\gamma$ receptor expression, which could partially explain the decrease in MHC class II expression observed (Supplemental Figure 6). Thus, the inflammatory monocytes induced by Poly-ICLC during M. tuberculosis infection may be impaired in both antigen presentation and IFN- $\gamma$-induced microbicidal activity, thus providing a possible explanation for the increased bacterial load seen in lungs of drug-treated animals.

Important evidence for the role of inflammatory monocytes in the Poly-ICLC-induced exacerbation of M. tuberculosis infection came from our discovery of the CCR2-dependent accumulation of this cell population in the lungs of drug-treated mice. Consistent with previous findings (22), Ccr2-deficient mice infected with M. tuberculosis displayed increased bacterial burdens compared with WT animals, which represents a complication in the interpretation of this experiment. Nevertheless, no further increase in bacterial load was observed in $\mathrm{Crr}^{-/-}$mice treated with PolyICLC, and the drug did not trigger the pronounced alterations in pulmonary myeloid cell populations seen in similarly treated WT animals. Moreover, lungs of drug-treated, infected WT mice displayed type I IFN-dependent increases in the levels of transcripts encoding CCL2, the major chemokine responsible for recruitment of circulating monocytes to sites of inflammation. Together, the above findings support a critical role for inflammatory monocytes in the Poly-ICLC-induced exacerbation of $M$. tuberculosis infection and suggest that these cells are recruited to the lungs by a CCR2-dependent mechanism rather than being differentiated locally as part of the pulmonary inflammatory response. Consistent with this hypothesis, elevations in the frequencies of $\mathrm{CD} 11 \mathrm{~b}^{+} \mathrm{Gr} 1^{\text {int }}$ cells were observed in the blood as well as the lungs of Poly-ICLC-treated, infected mice but not in spleen or mediastinal lymph nodes (data not shown). 

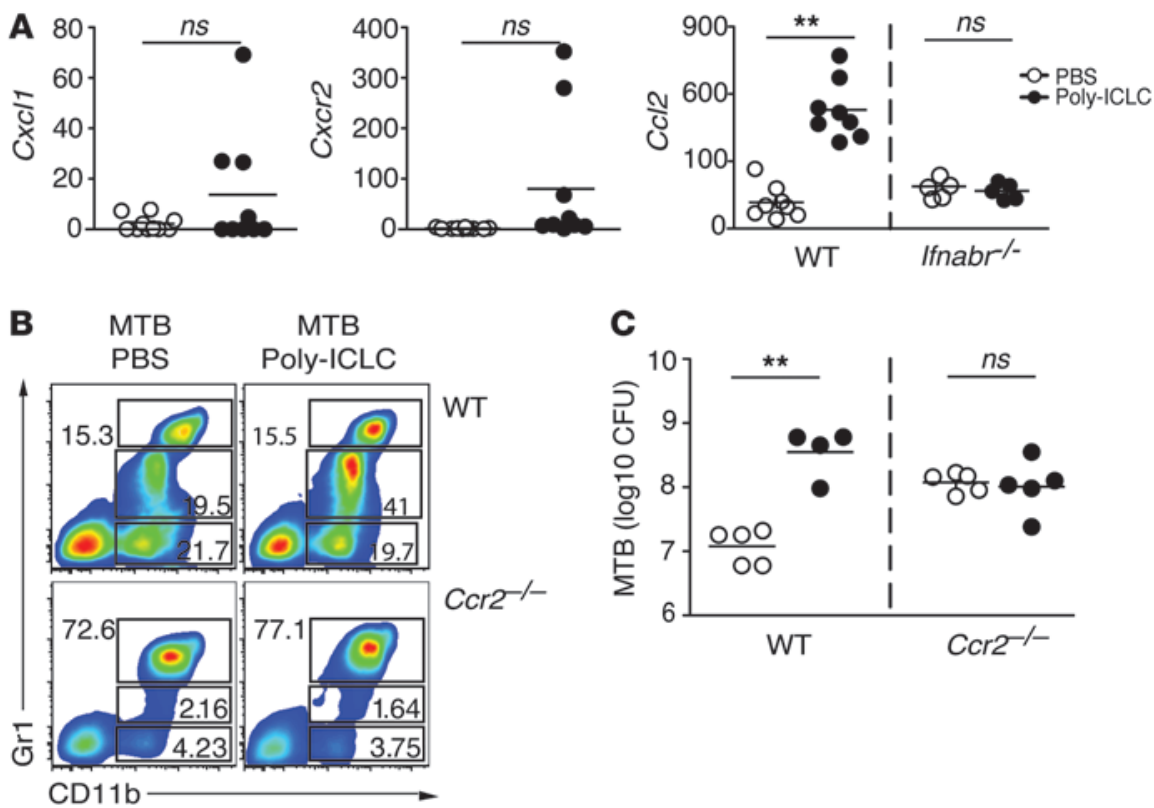

Figure 7

Both recruitment of $C D 11 \mathrm{~b}+\mathrm{Gr} 1$ int cells and exacerbation of $M$. tuberculosis infection after PolyICLC treatment depend on CCR2 expression. (A) Cxc/1, Cxcr2, and Cc/2 mRNA expression levels, determined by real-time PCR in the lungs of $M$. tuberculosis-infected animals treated with PBS (open circles) and Poly-ICLC (filled circles). The results shown represent the fold increase relative to that observed in untreated, naive mice, with circles representing individual mice. Data were pooled from 3 independent experiments with similar results. (B) Representative flow cytometry dot plots of $C D 11 b+G r 1^{-}, C D 11 b+G r 1^{\text {int }}$, and $C D 11 b+G r 1$ high pulmonary leukocytes isolated from Poly-ICLC- or PBS-treated, M. tuberculosis-infected WT mice or Ccr2-deficient animals. Numbers within or beside each box refer to the frequencies of cells within each gate. (C) Pulmonary mycobacterial loads in PBS-treated (open circles) and Poly-ICLC-treated (closed circles) WT and Ccr2-deficient mice. Circles represent individual animals. The data shown are representative of 3 independent experiments. (A and $\mathbf{C})$ Horizontal lines represent comparisons between Poly-ICLC- and PBS-treated, M. tuberculosis-infected mice. ${ }^{\star \star} P<0.01$.

In addition to exacerbating murine tuberculosis when given at the time of initial pathogen exposure, intranasally administrated PolyICLC promoted bacterial growth and host mortality when initiated during chronic infection. The latter observation suggests that dysregulated type I IFN production can trigger $M$. tuberculosis growth and enhance disease in the setting of an established cellular immune response to the pathogen and thus has the potential to reactivate latent infection, despite previous studies reporting beneficial effects of these cytokines on patients with tuberculosis when combined with chemotherapy $(37,38)$. In the case of Poly-ICLC, it has not yet been determined whether these effects are a direct outcome of the intranasal route of administration of the drug, although this seems unlikely, since systemic effects were also observed on the circulating monocyte pool in the same experiments (data not shown).

We are unaware of clinical studies documenting reactivation of latent tuberculosis in individuals given type I IFN therapy, as seen in individuals given TNF- $\alpha$ inhibitors (39). However, adverse pulmonary effects, such as pneumonitis, have been described in some patients receiving type I IFN treatment, who require cessation of treatment and/or administration of corticosteroids to resolve their symptoms (e.g., refs. 40, 41). Even less is known about the effects of type I IFN inducers, such as Poly-ICLC, on latent tuberculosis, since the use of these compounds has been limited to clinical trials. Nevertheless, because of the promising effects seen with type I IFN inducers in immunotherapy of both cancer and viral infection and as adjuvants for vaccination (42-44), major new clinical applications for these drugs are likely to emerge. While the data presented here are confined to a specific murine experimental model of tuberculosis, they suggest that reactivation of latent M. tuberculosis should be evaluated as a possible side effect when testing type I IFN inducers or type I IFN treatment itself in clinical settings in which the treated population is heavily exposed and therefore at risk of tuberculosis.

\section{Methods}

Mice. WT C57BL/ 6 and Ifnabr-deficient mice, on a B6 background, were purchased from Taconic Farms under the NIAID Animal Supply Contract. Ccr2-deficient mice were obtained from The Jackson Laboratory. Il13ra1 mice were provided by Thirumalai Ramalingan (NIAID) and Tom Wynn (NIAID). All mice were maintained in specific pathogen-free conditions at an American Association of Laboratory Animal Careaccredited BioSafety Level 3 containment facility at the NIAID, under a study proposal approved by the NIAID Animal Care and Use Committee. Male and female mice between 8 and 12 weeks of age were used in all experiments.

M. tuberculosis infection and measurement of bacterial loads. M. tuberculosis $\mathrm{H} 37 \mathrm{Rv}$ bacilli harvested from infected mouse lungs were expanded to log phase in Middlebrook 7H9 broth, supplemented with oleic acid-albumin-dextrose-catalase (Difco), quantified on 7H11 agar plates, and stored in aliquots at $-80^{\circ} \mathrm{C}$. Mice were exposed to bacteria via the aerosol route, using a nose-only exposure unit ( $\mathrm{CH}$ Technologies). Each mouse received approximately 100 CFUs of $M$. tuberculosis. Bacterial loads in various organs were measured by culture on 7H11 agar plates (Difco).

Poly-ICLC and in vivo treatment. Poly-ICLC (Hiltonol), a synthetic analogue of viral dsRNA, was supplied by Oncovir Inc. Mice were inoculated intranasally with Poly-ICLC ( $6 \mu \mathrm{g}$ in $50 \mu \mathrm{l} /$ mouse) twice weekly for 28 days, starting 1 day or 4 months after $M$. tuberculosis exposure. Control animals received an equivalent volume of PBS.

Isolation of pulmonary lenkocytes. Lungs were perfused through the heart with PBS before excision, incubated with $0.4 \mathrm{mg} / \mathrm{ml}$ Liberase $\mathrm{CI}$ solution (Roche Biochemicals) for 30 minutes at $37^{\circ} \mathrm{C}$, and minced after a small portion had been saved for pathology and RNA extraction. Single-cell suspensions were prepared by passing tissue through a $100-\mu \mathrm{m}$ nylon cell strainer and an aliquot saved for CFU measurement. Lung cells were centrifuged in 35\% Percoll (Pharmacia Biotech) for 15 minutes at $700 \mathrm{~g}$ to enrich pulmonary leukocytes. Supernatants were decanted, the cell pellets were resuspended, and erythrocytes were lysed using ACK lysing buffer (BioWhittaker).

Flow cytometric analysis of cell populations and intracellular cytokine production. Freshly isolated lung cells were analyzed ex vivo or after culture for 6 hours with medium alone, PPD $(5 \mu \mathrm{g} / \mathrm{ml})$, or anti-CD3 $(1 \mu \mathrm{g} / \mathrm{ml})$. During the last 4 hours of culture, brefeldin A and monensin $(1 \mu \mathrm{g} / \mathrm{ml})$ were added before staining for surface markers. To analyze the phenotype of pulmonary subpopulations, cell suspensions were stained with $\mathrm{mAb}$ specific for CD3-FITC, 
CD8-APC-Alexa Fluor 750, CD44-FITC, CD44-APC-Alexa Fluor 750, B220FITC, CD19-PE, CD11b-APC, MAC-3-PE, MHC-II-biotin, CD119 (IFN- $\gamma$ R1) biotin, and Annexin V-FITC (all from BD Biosciences - Pharmingen); Gr-1-PECy5, CD115-APC, CD11c-Alexa Fluor 700, F4/80-Pacific blue, and CD11b-PECy7 (all from eBioscience); and CD4-Pacific orange (Caltag). Suspensions were then fixed and permeabilized according to the kit instructions (Fix/Perm kit; BD Biosciences).

Intracellular cytokine staining was performed using $\mathrm{mAb}$ specific for IFN- $\gamma$ (PECy7 labeled), TNF- $\alpha$ (FITC or Pacific blue labeled) (eBioscience), and iNOS (FITC) (BD Biosciences - Pharmingen). Quantum dot 605-conjugated streptavidin (Invitrogen) was added during intracellular staining. Data were collected using a LSR II (BD Biosciences - Immunocytometry Systems) with Diva (BD Biosciences) and analyzed with FlowJo (Tree Star) software. The expression of myeloid markers was analyzed after $\mathrm{CD}^{+} \mathrm{T}$ cells exclusion.

Purification of pulmonary myeloid subpopulations. After isolation of pulmonary leukocytes, the myeloid $\mathrm{CD} 11 \mathrm{~b}^{+} \mathrm{Gr} 1^{-}$and $\mathrm{CD} 11 \mathrm{~b}^{+} \mathrm{Gr} 1^{\text {int }}$ cell subpopulations from $M$. tuberculosis-infected animals were sorted with a FACSAria cell sorter (BD Biosciences), using the combination of antibodies described above. The purity of each cell population is shown in Supplemental Figure 1. $\mathrm{CD} 11 \mathrm{~b}^{+} \mathrm{Gr} 1^{-}$and $\mathrm{CD} 11 \mathrm{~b}^{+} \mathrm{Gr} 1^{\text {int }}$ cells were then seeded on $7 \mathrm{H} 11$ agar plates (Difco), and bacterial loads were measured after 3 to 4 weeks.

Quantitative reverse-transcription PCR. RNA was isolated from lung samples with the RNeasy kit (Qiagen) or TRIzOL (Sigma-Aldrich). Oligo(dT) primers and M-MLV-Reverse Transcriptase (Promega) were used to synthesize first-strand cDNA from $1 \mu \mathrm{g}$ total RNA. Real-time PCR in an ABI Prism 7900 Sequence Detection System (Applied Biosystems) was performed with SYBR Green PCR Master Mix (Applied Biosystems). Data were generated by the comparative threshold cycle $(\lambda \mathrm{CT})$ method, by normalizing to hypoxanthine guanine phosphoribosyltransferase (HPRT), and expressed as a fold change compared with uninfected controls. The primer sequences for the genes examined in the study were published elsewhere (45) or designed using PrimerExpress software (Applied Biosystems) and are listed as follows: IFN- $\beta$, forward, AGCTCCAAGAAAGGACGAACA, reverse, GCCCTGTAGTGTAGGTTGATC; CXCR2, forward, GGTGGGGAGTTCGTGTAGAA, reverse, CGAGGTGCTAGGATTTGAGC; CXCL1, forward, TTTTGTATGTATTAGGGTGAGGACAT, reverse, GCGTGTTGACCATACAATATGAA; CCL2, forward, ACTGAAGCCAGCTCTCTCTTCCTC, reverse, TTCCTTCTTGGGGTCAGCACAGAC.

$I F N-\beta$ production assay. Lungs were perfused through the heart with PBS before excision, chopped, and homogenized in $1 \mathrm{ml}$ PBS $(0.4 \mathrm{M}$ $\mathrm{NaCl}$ and $\left.10 \mathrm{mM} \mathrm{NaPO}_{4}\right)$ containing antiproteases $(0.1 \mathrm{mM}$ phenyl- methylsulfonyl fluoride, $0.1 \mathrm{mM}$ benzethonium chloride, $10 \mathrm{mM}$ EDTA, and $20 \mathrm{KI}$ aprotinin A) and $0.05 \%$ Tween 20 . The samples were then centrifuged for 10 minutes at $3,000 \mathrm{~g}$, and IFN- $\beta$ levels were determined in the supernatants using ELISA (PBL InterferonSource) according to the manufacturer's recommendation.

Histopathology. Tissues were fixed in $10 \%$ buffered formalin, sectioned at $5 \mu \mathrm{m}$, and stained with $\mathrm{H} \& \mathrm{E}$ or by the Ziehl-Neelsen method to detect acidfast mycobacteria. The extent of pulmonary inflammation was determined by light microscopy at magnifications of $\times 25, \times 50, \times 200$, and $\times 400$. The degree of necrosis was assessed using a scale of $1-5$ (with 5 representing the most severe necrotic response). All histological analyses were performed by a pathologist on blinded specimens without knowledge of the experimental groups. The lesions on 1 lung section were scored for each animal.

Statistics. ANOVA was used to assess the statistical significance of differences in the data means in experiments involving multiple groups. Student's $t$ test was used in experiments involving pairwise comparisons. A double “**” indicates a $P$ value of less than 0.01 , while a single “*” indicates $0.01<P$ value $<0.05$. $P$ values of less than 0.05 were considered significant.

\section{Acknowledgments}

We are grateful to Sara Hieny, Pat Caspar, Sandy White, and Robert Thompson for excellent technical assistance and to the NIAID Flow Cytometry Section, particularly Bishop Hague for performing the FACS cell purifications and Kevin Holmes and David Stephany for their advice on multicolor flow cytometry. We also thank Stefanie Vogel and Brian Kelsall for their valuable advice and Siamon Gordon, Dragana Jankovic, Tom Wynn, and Giorgio Trinchieri for their helpful comments on this manuscript. This study was supported by the Intramural Research Program of NIAID, NIH, Department of Health and Human Services.

Received for publication August 13, 2009, and accepted in revised form February 17, 2010.

Address correspondence to: Lis R.V. Antonelli, Centro de Pesquisas René Rachou - FIOCRUZ - Av. Augusto de Lima, 1715, Belo Horizonte, MG, Brazil, 30190-002. Phone: 55.31.3379.7766; Fax: 55.31.3349.7835; E-mail: lisantonelli@cpqrr.fiocruz.br.

Lis R.V. Antonelli's present address is: Centro de Pesquisas René Rachou - FIOCRUZ, Belo Horizonte, Minas Gerais, Brazil.
1. Isaacs A, Lindenmann J. Virus interference. I. The interferon. Proc R Soc Lond B Biol Sci. 1957; 147(927):258-267.

2. Nakane A, Minagawa T. Alternative induction of IFN-alpha and IFN-gamma by Listeria monocytogenes in human peripheral blood mononuclear leukocyte cultures. J Immunol. 1981;126(6):2139-2142.

3. Diefenbach A, et al. Type 1 interferon (IFNalpha/ beta) and type 2 nitric oxide synthase regulate the innate immune response to a protozoan parasite. Immunity. 1998;8(1):77-87.

4. Rodel J, Groh A, Vogelsang H, Lehmann M, Hartmann M, Straube E. Beta interferon is produced by Chlamydia trachomatis-infected fibroblast-like synoviocytes and inhibits gamma interferoninduced HLA-DR expression. Infect Immun. 1998; 66(9):4491-4495.

5 . Bogdan C. The function of type I interferons in antimicrobial immunity. Curr Opin Immunol. 2000; 12(4):419-424.

6. McRae BL, Semnani RT, Hayes MP, van Seventer GA. Type I IFNs inhibit human dendritic cell IL-12 production and Th1 cell development. J Immunol. 1998;160(9):4298-4304.
7. Petricoin EF 3rd, et al. Antiproliferative action of interferon-alpha requires components of T-cell-receptor signalling. Nature. 1997;390(6660):629-632.

8. O'Connell RM, et al. Type I interferon production enhances susceptibility to Listeria monocytogenes infection. J Exp Med. 2004;200(4):437-445.

9. Carrero JA, Calderon B, Unanue ER. Type I interferon sensitizes lymphocytes to apoptosis and reduces resistance to Listeria infection. J Exp Med. 2004; 200(4):535-540.

10. Denis M. Recombinant murine beta interferon enhances resistance of mice to systemic Mycobacterium avium infection. Infect Immun. 1991;59(5):1857-1859.

11. Manca C, et al. Virulence of a Mycobacterium tuberculosis clinical isolate in mice is determined by failure to induce Th1 type immunity and is associated with induction of IFN-alpha /beta. Proc Natl Acad Sci US A. 2001;98(10):5752-5757.

12. Manca C, et al. Hypervirulent M. tuberculosis W/Beijing strains upregulate type I IFNs and increase expression of negative regulators of the Jak-Stat pathway. J Interferon Cytokine Res. 2005;25(11):694-701.

13. Ordway D, et al. The hypervirulent Mycobacterium tuberculosis strain HN878 induces a potent TH1 response followed by rapid down-regulation. J Immunol. 2007;179(1):522-531.

14. Kujawski LA, Talpaz M. The role of interferonalpha in the treatment of chronic myeloid leukemia. Cytokine Growth Factor Rev. 2007;18(5-6):459-471.

15. Borden EC, et al. Interferons at age 50: past, current and future impact on biomedicine. Nat Rev Drug Discov. 2007;6(12):975-990.

16. Levy HB, Riley FL, Lvovsky E, Stephen EE. Interferon induction in primates by stabilized polyriboinosinic acid-polyribocytidylic acid: effect of component size. Infect Immun. 1981;34(2):416-421.

17. Levy HB, et al. A modified polyriboinosinic-polyribocytidylic acid complex that induces interferon in primates. J Infect Dis. 1975;132(4):434-439.

18. Harris J, et al. Thelper 2 cytokines inhibit autophagic control of intracellular Mycobacterium tuberculosis. Immunity. 2007;27(3):505-517.

19. Schreiber T, et al. Autocrine IL-10 induces hallmarks of alternative activation in macrophages and suppresses antituberculosis effector mechanisms without compromising $\mathrm{T}$ cell immunity. Jimmunol. 2009;183(2):1301-1312.

20. Serbina NV, Pamer EG. Monocyte emigration from 
bone marrow during bacterial infection requires signals mediated by chemokine receptor CCR2. Nat Immunol. 2006;7(3):311-317.

21. Kurihara T, Warr G, Loy J, Bravo R. Defects in macrophage recruitment and host defense in mice lacking the CCR2 chemokine receptor. J Exp Med. 1997;186(10):1757-1762.

22. Peters W, Scott HM, Chambers HF, Flynn JL, Charo IF, Ernst JD. Chemokine receptor 2 serves an early and essential role in resistance to $\mathrm{Myco}$ bacterium tuberculosis. Proc Natl Acad Sci U S A. 2001; 98(14):7958-7963

23. Cooper AM, Dalton DK, Stewart TA, Griffin JP, Russell DG, Orme IM. Disseminated tuberculosis in interferon gamma gene-disrupted mice. J Exp Med. 1993;178(6):2243-2247.

24. Flynn JL, Chan J, Triebold KJ, Dalton DK, Stewart TA, Bloom BR. An essential role for interferon gamma in resistance to Mycobacterium tuberculosis infection. J Exp Med. 1993;178(6):2249-2254.

25. Flynn JL, et al. Tumor necrosis factor-alpha is required in the protective immune response against Mycobacterium tuberculosis in mice. Immunity. 1995; 2(6):561-572.

26. Davis JM, Ramakrishnan L. The role of the granuloma in expansion and dissemination of early tuberculous infection. Cell. 2009;136(1):37-49.

27. Bold TD, Ernst JD. Who benefits from granulomas, mycobacteria or host? Cell. 2009;136(1):17-19.

28. Stockinger S, et al. Production of type I IFN sensitizes macrophages to cell death induced by Listerid monocytogenes. J Immunol. 2002;169(11):6522-6529.

29. Dunay IR, et al. Gr1(+) inflammatory monocytes are required for mucosal resistance to the pathogen Toxoplasma gondii. Immunity. 2008;29(2):306-317.

30. Serbina NV, Salazar-Mather TP, Biron CA, Kuziel WA, Pamer EG. TNF/iNOS-producing dendritic cells mediate innate immune defense against bacterial infection. Immunity. 2003;19(1):59-70.

31. Movahedi K, et al. Identification of discrete tumorinduced myeloid-derived suppressor cell subpopulations with distinct $\mathrm{T}$ cell-suppressive activity. Blood. 2008;111(8):4233-4244.

32. Huang B, et al. Gr-1+CD115+ immature myeloid suppressor cells mediate the development of tumorinduced $T$ regulatory cells and T-cell anergy in tumorbearing host. Cancer Res. 2006;66(2):1123-1131.

33. Geissmann F, et al. Blood monocytes: distinct subsets, how they relate to dendritic cells, and their possible roles in the regulation of T-cell responses. Immunol Cell Biol. 2008;86(5):398-408.

34. Skold M, Behar SM. Tuberculosis triggers a tissuedependent program of differentiation and acquisition of effector functions by circulating monocytes. J Immunol. 2008;181(9):6349-6360.

35. Geiss G, Jin G, Guo J, Bumgarner R, Katze MG, Sen GC. A comprehensive view of regulation of gene expression by double-stranded RNA-mediated cell signaling. J Biol Chem. 2001;276(32):30178-30182.

36. Ling PD, Warren MK, Vogel SN. Antagonistic effect of interferon-beta on the interferon-gammainduced expression of Ia antigen in murine macrophages. J Immunol. 1985;135(3):1857-1863.

37. Giosue S, et al. Effects of aerosolized interferonalpha in patients with pulmonary tuberculosis. Am J Respir Crit Care Med. 1998;158(4):1156-1162.
38. Giosue S, et al. Aerosolized interferon-alpha treatment in patients with multi-drug-resistant pulmonary tuberculosis. Eur Cytokine Netw. 2000;11(1):99-104.

39. Keane J, et al. Tuberculosis associated with infliximab, a tumor necrosis factor alpha-neutralizing agent. N Engl J Med. 2001;345(15):1098-1104.

40. Carrillo-Esper R, Gonzalez-Avila D, Uribe-Rios M, Mendez-Sanchez N. Interstitial pneumonitis associated with pegylated interferon alpha- $2 \mathrm{~b}$ therapy for chronic hepatitis C: case report. Ann Hepatol. 2008;7(1):87-90.

41. Trullas Vila JC, Padilla Lopez DR, Bisbe Company V, Soler Simon S, Cortes Hausmann P, Bisbe Company J. [Organizing pneumonia associated with the use of pegylated interferon alfa]. Arch Bronconeumol. 2008;44(3):173-174.

42. Trumpfheller C, et al. The microbial mimic poly IC induces durable and protective CD4+ T cell immunity together with a dendritic cell targeted vaccine. Proc Natl Acad Sci U S A. 2008;105(7):2574-2579.

43. Stahl-Hennig C, et al. Synthetic double-stranded RNAs are adjuvants for the induction of T helper 1 and humoral immune responses to human papillomavirus in rhesus macaques. PLoS Pathog. 2009; 5(4):e1000373.

44. Longhi MP, et al. Dendritic cells require a systemic type I interferon response to mature and induce CD4+ Th1 immunity with poly IC as adjuvant. J Exp Med. 2009;206(7):1589-1602.

45. Gautier G, et al. A type I interferon autocrine-paracrine loop is involved in Toll-like receptor-induced interleukin-12p70 secretion by dendritic cells. J Exp Med. 2005;201(9):1435-1446. 